\title{
LA EDUCACIÓN COMO FUNDAMENTO DEL (MEJOR) RÉGIMEN POLÍTICO EN ARISTÓTELES
}

\author{
EDUCATION AS THE FOUNDATION OF \\ ARISTOTLE'S (BEST) POLITICAL REGIME
}

\author{
VIVIANA SUÑOL* \\ Universidad Nacional de La Plata \\ Consejo Nacional de Investigaciones Científicas y Técnicas
}

Resumen: Tradicionalmente se le otorgó a Aristóteles un lugar secundario en la historia de la educación griega clásica. A pesar del florecimiento del estudio de su teoría de la educación en los últimos treinta años, aún hoy no se reconoce de manera suficiente la importancia que este tema tiene en su pensamiento (DESTRÉE 2013). La educación es la piedra fundamental de la ciencia (ético)-política aristotélica, pues a partir de ella se construye y se sostiene todo régimen político (Política VIII 1), a la vez que resulta indispensable para que el hombre complete su naturaleza (Pol. VII 17 1337a1-3). El propósito del presente trabajo es mostrar que a pesar del carácter inconcluso de su tratamiento del tema, Aristóteles nos ofrece en Pol. VII 13-17 y VIII 1-2 elementos suficientes para reconocer la relevancia filosófica de la educación e identificar los aspectos más importantes de su teoría educacional.

Palabras Clave: Aristóteles, Educación, Política, Ética, Régimen ideal.

AвSTRACT: Aristotle has traditionally occupied a secondary role in the history of classical Greek education. Despite the fact that the study of the theory of education has flourished in the last thirty years, the importance of this issue in his thought still seems not to be sufficiently recognized (DesTréE 2013). Education is the cornerstone of Aristotelian (ethical)-political science, since the construction and preservation of any

\footnotetext{
* Investigadora Asistente del CONICET en el Instituto de Investigaciones en Humanidades y Ciencias Sociales de la Facultad de Humanidades y Ciencias de la Educación de la Universidad Nacional de La Plata, Argentina. Dirección electrónica: vsunyol@gmail.com
} 
political regime and not only the ideal one, depends on it (Politics VIII 1), while it is essential for men to complete their nature (Politics VII 17 1337a1-3). The purpose of this paper is to show that, despite the apparently inconclusive nature of his treatment of the subject, in Politics VII 13-17 and VIII 1-2 Aristotle provides enough information to recognize the philosophical relevance of education and identify the most important aspects of his educational theory.

KeYwords: Aristotle, Education, Politics, Ethics, Ideal Regime.

\section{Introducción}

Hasta mediados del siglo xx la literatura especializada (v.gr. JAeger 2011, Marrou 1948) le otorgó a Aristóteles un lugar secundario en la historia de la educación griega clásica, debido -entre otras razones- al compromiso que tradicionalmente se le atribuyó con los modelos educativos de sus dos grandes antecesores, i.e. Platón e Isócrates (DL V 3, 3-4) $)^{1}$ y a la aparente ausencia de un tratamiento sistemático del tema en el corpus, la cual se reflejaría en la menor frecuencia de empleo del vocabulario educativo (Tachibana, 2012, 36-41). A pesar de la recuperación del interés por la teoría aristotélica de la educación tras la Segunda Guerra Mundial y del posterior florecimiento de su estudio en la década del ochenta, ${ }^{2}$ aún hoy parece no reconocerse de manera suficiente la importancia que la educación, en general, y la música, en particular, tienen en el pensamiento ético-político aristotélico. ${ }^{3}$ Tal como advierte Destrée $(2013,301)$ : “desde el punto de vista de Aristóteles (que probablemente es contrario al nuestro) este tema es absolutamente central a su proyecto". ${ }^{4}$ Algunos autores sostienen que la educación es parte de la política (ReEve, 1998, 49), otros aseguran que la ética y la educación se funden una con la otra (Hummel, 1997, 2). A mi entender,

1 El testimonio de Diógenes Laercio abona la idea de la falta de originalidad del modelo educativo aristotélico, puesto que su labor pedagógica conjuga los métodos de sus antecesores: "kaì pròs thésin sunegúmnaze toùs mathêtás, háma kai rẹtorikôos epaskônn".

2 Para la evolución histórica de los estudios acerca de la teoría aristotélica de la educación, cfr. Tachibana (2012).

3 Ya en su libro de 1982, Lord afirmaba: "The discussion of music and education in the final books of the Politics is virtually the only account of those subjects in the extant writings of Aristotle or Peripatetic School, and it is perhaps the most comprehensive and systematic account surviving from antiquity. And yet this discussion -of critical importance for the study both classical thought and of classical poetics- has not, I believe, been adequately served by historical and philological analysis or by interpretation". Ibid., 24. Las cursivas son mías.

4 "But from Aristotle's point of view (and, perhaps, contrary to ours) this theme is absolutely central to his project." 
la educación es la piedra fundamental de la ciencia (ético)-política aristotélica, pues a partir de ella se construye y se mantiene no solo el régimen ideal, sino todo régimen político (Pol. VIII 1), a la vez que resulta indispensable para que el hombre complete su naturaleza (Pol. VII 17 1337a1-3). ${ }^{5}$ Por esta razón, no es posible estudiar la educación como un tema independiente, sino como parte esencial de la ética y de la política o, más precisamente, como fundamento de la ciencia que las conjuga.

Una de las principales dificultades que plantea el estudio de la educación en la obra de Aristóteles es que el último libro de la Política, que está enteramente dedicado a la educación de los jóvenes en el régimen ideal, nos ha llegado incompleto (Pol. VIII 7 1342b 33-34). Esto ha llevado a algunos intérpretes a sostener que el tratamiento del tema que allí ofrece no es más que una mera discusión preliminar, la cual resulta insuficiente para responder a las preguntas esenciales sobre los fines y medios de la educación, y que obliga a reconstruir la perspectiva aristotélica a partir de lo dicho en los dos últimos libros de la Pol. y de lo que puede extraerse de otros textos (ReEve, 1998, 49). ${ }^{6}$ Asimismo, se pretende establecer una suerte de escisión conceptual entre la descripción del modelo educativo del régimen ideal, la cual constituiría una teoría de la educación en un sentido genuino y la teoría de la educación moral, que sería posible reconstruir a partir de sus distintas obras (Reeve, 1998, 58; Tachibana, 2012, 61). ${ }^{7}$ Sin embargo, Aristóteles se dedica ampliamente al estudio de la educación en Pol. VII-VIII y su diseño curricular está íntimamente imbricado a la instrucción moral en todas y cada una de las disciplinas propuestas, siendo paradigmático el caso de la mousiké. En definitiva, aun cuando el libro VIII está efectivamente incompleto, el Estagirita nos ofrece elementos suficientes para reconocer la

5 "pâsa gàr téchne kai paideía tò prosleîpon boúletai tês phúseos anapleroûn."

6 Reeve intenta dar cuenta de este carácter preliminar de la discusión remitiendo a Pol. 1336b24-27, en donde Aristóteles reconoce que hace un tratamiento somero y promete hacer una consideración más detallada posteriormente. Aun cuando no es posible hallar este desarrollo ulterior en los manuscritos de la Pol., es importante advertir que el Estagirita refiere allí a la cuestión de la exposición de los jóvenes a los yambos y comedias, y no al tema de la educación en general (Kraut 1997, 165 ad loc.). En consecuencia, esta advertencia no respalda la idea de un tratamiento superficial de la educación en la Pol.

7 Esto se evidencia en el trabajo de Reeve, quien luego de estudiar los propósitos de la educación aristotélica y antes de comenzar a concentrarse en sus medios asegura: "It will be well now to start over, ignoring the distinction between training, habituation, and instruction and their different targets in the individual soul, and focusing instead on the actual educational institutions and practices Aristotle recommends.” Por su parte, Tachibana (2012, 61) distingue entre el estudio de la teoría aristotélica de educación en un sentido genuino, i.e., el currículo, y su teoría de la educación moral. 
relevancia filosófica que la educación tiene en su pensamiento ético-político e identificar los aspectos más importantes de su teoría educacional. ${ }^{8}$ De hecho, su programa pedagógico revela que no es posible entender la teoría aristotélica de la educación si no es como una teoría de la educación moral. El propósito del presente trabajo es reconocer la importancia de la educación en su pensamiento y, en particular, investigar el papel que Aristóteles le otorga en la construcción del mejor régimen político mediante el análisis de los capítulos en los que el filósofo plantea y desarrolla el tema, i.e. Pol. VII 13-17 y también VIII 1-2, donde hace consideraciones pedagógicas generales que se aplican a todo régimen. Por razones de extensión, no ahondaremos aquí en el diseño curricular que propone para la educación pública ni en la función esencial que la mousiké cumple en este proyecto político, i.e. Pol. VIII 3-7, cuestión que -a mi entender- requiere un estudio específico.

\section{El papel de la educación en la construcción del mejor régimen}

Luego de insistir en los tres primeros capítulos de Pol. VII que la cuestión política del mejor régimen es inseparable de la pregunta ética por la vida mejor (Pol. VII 1-3) y de analizar en los capítulos siguientes las condiciones geográficas y sociales de dicho régimen (Pol. VII 4-12), ${ }^{10}$ en Pol. VII 13 retoma la consideración acerca de la eudaimonía, reiterando la idea de que es el fin y proponiendo tácitamente a la educación como el medio que el legislador dispone para alcanzarla. ${ }^{11}$ En este contexto, Aristóteles plantea la pregunta fundacional de su reflexión sobre la educación: cómo el hombre deviene virtuoso (pồs anèr gínetai spoudaîos, Pol. 1332a35-36), a cuya respuesta se aboca en lo que resta del libro y le dedica enteramente el último. Aristóteles establece aquí una relación instrumental entre educación y eudaimonía, y comienza a analizar el modelo

\footnotetext{
8 "Although it appears that the discussion is not completed in the text that is extant, there is still a good deal of material to work with." Nightingale (1996, 35). Las cursivas son mías.

9 Para el análisis de esta cuestión, cfr. Suñol (2013, 24-31) y (2014).

10 Aristóteles anticipa, en cierto modo, la indagación posterior sobre la educación cuando en Pol. VII 2 (1325a7-10) asegura que "es propio del legislador noble investigar (theásasthai) cómo la ciudad, el género de los hombres y toda otra comunidad participan de la vida buena y de la felicidad que es posible para ellos".

11 Según el filósofo, el hecho de que la ciudad sea virtuosa es la resultante de la virtud de cada uno de sus ciudadanos y no del conjunto, puesto que todos participan del régimen mejor. Kraut $(1997,130-131)$ asegura que no está claro si Aristóteles está haciendo una afirmación fuerte de que cada uno de los ciudadanos debe ser excelente o una más débil, que afecta a solo una parte, si bien luego reconoce que la educación: "will make all of them virtuous".
} 
educativo que permite a los ciudadanos alcanzar esta última (KRAUT, 1997, 123 ad 1331b 24-26). En primer lugar, señala que los hombres se hacen buenos (agathoi) y virtuosos (spoudaîoi) por medio de la naturaleza, el carácter y la razón (Pol. 1332a39-40), ${ }^{12}$ y establece la necesidad de la armonización de estos tres factores entre sí (1332b5-6). Remitiendo a Pol. VII 7 (1327a36-38), donde establece que la naturaleza mejor de los ciudadanos es la que conjuga inteligencia y coraje, ${ }^{13}$ aquí afirma que solo los hábitos y la razón son tarea de la educación (tò dè loipòn érgon éde paideías, Pol. 1332b10). ${ }^{14}$

En Pol. VII 14 es donde con más claridad se observa la interrelación o, más precisamente, la unidad entre política, ética y educación. La estructura misma del capítulo da cuenta de la íntima conexión que existe entre ellas, pues en primer lugar (Pol.1332b11-1333a15), Aristóteles analiza las razones que justifican un régimen de alternancia entre gobernantes y gobernados; luego (Pol. 1333a161333b5), introduce la división del alma y formula el célebre principio teleológico conforme al cual, la guerra, el trabajo y las acciones necesarias son con vistas a la paz, el ocio y las acciones nobles, respectivamente; por último (Pol.1333b51334a10), hace una extensa crítica al régimen espartano, en especial, a su sistema educativo. Cada una de las partes del capítulo fundamenta y explica la siguiente, siendo la educación el punto en el que todas convergen. Por esta razón, es importante analizar las distintas referencias que Aristóteles hace a ella a lo largo de este capítulo medular. Desde un comienzo, establece que la educación debe corresponderse (akoloutheîn) con el vínculo que en cada régimen existe entre gobernantes y gobernados (Pol. 1332b15-16). Puesto que para el mejor régimen propone la alternancia, asegura que es necesario que la educación de ambos sea la misma y, a su vez, distinta en función del rol que los ciudadanos desempeñan en cada momento (Pol. 1332b42-1333a2). En este sentido, afirma que ser gobernado

12 La referencia a los tres medios necesarios para que los hombres se hagan buenos también puede encontrarse en EN 1179b20-21 y en EE I 1 1214a14-19. En el primer caso, los términos que Aristóteles emplea son naturaleza, hábito y enseñanza (hoì mèn phúsei hoì d'éthei hoì dè didachêi $i$ y, en el segundo, naturaleza (phúsis), práctica (áskesis), y aprendizaje (máthesisi).

13 A pesar de que en Pol. VII 13 sitúa a la naturaleza fuera de la competencia del legislador (hoúto kaì poión tina tò sôma kaì tẹn psuchẹn, Pol. 1341a41-42), el programa educativo aristotélico regula la constitución del cuerpo de los ciudadanos (Pol. VII 16 y 17). En este sentido, la tarea del legislador abarca también el ámbito natural.

14 Teniendo en cuenta el lugar que le otorga a la música en su programa pedagógico, resulta significativo que para aludir a la educación de la razón emplee el participio del verbo akoúo: "pues aprendemos unas cosas habituándonos y otras, escuchando (akoúontes)" (Pol. 1332b10-11). 
es condición para poder gobernar (Pol. 1333a2-3). ${ }^{15}$ Como advierte BÉNatouïL (2011, 158, 160-161), además del condicionamiento y del intercambio generacional, entre ambas funciones hay también un vínculo pedagógico. ${ }^{16}$

En las restantes referencias que Aristóteles hace a la educación a lo largo del capítulo, la presenta como responsabilidad del legislador, sugiriendo que de ella depende la naturaleza y la pervivencia de los regímenes en general, tal como lo refleja el caso de Lacedemonia (Pol. 1334a9-10). ${ }^{17}$ La identidad que establece entre la virtud del ciudadano, la del gobernante y la del hombre mejor (Pol. 1333a11-13) solo es posible en el régimen ideal. ${ }^{18}$ De ahí que proponga que gobernantes y gobernados sean los mismos y, a su vez, distintos, lo cual implica el cambio intergeneracional. En este mismo sentido, reitera y amplía la pregunta formulada en el capítulo anterior, al plantear que el legislador debe ocuparse de cómo los hombres se hacen buenos (hópos ándres agathoi gígnontai), por medio de qué hábitos (epitedeumáton) y cuál es el fin de la vida mejor (Pol. 1333a14-16). La enunciación misma muestra que para Aristóteles el análisis de la educación (referido en las dos primeras preguntas) es inseparable de la reflexión ética sobre la vida más elegible (aludida en la última) y que, a su vez, ambos son

15 Aristóteles alude a esta condición en Pol. III 4 1277b9-13 en el marco de su definición de la dominación política: "[...] el gobernante debe aprender siendo gobernado, como se aprende a ser jefe de caballería habiendo servido en ella, y general de infantería sirviendo a las órdenes de otros y habiendo sido jefe de regimiento, y jefe de compañía."

16 Kraut $(1997,137)$ asegura que en Pol. VII 14 Aristóteles no especifica en qué se diferencia la educación del gobernante, en la medida en que esta no consiste meramente en la instancia preliminar de ser gobernado: "one cannot learn how to be a general merely by learning how to take orders; one must learn something about strategy." Por el contrario, BÉNATOü̈L subraya la finalidad pedagógica de la función subordinada, pues constituye una etapa de perfeccionamiento de las disposiciones de los jóvenes ciudadanos en la que aprenden la virtud política.

17 La primera referencia que hace en el capítulo a la educación muestra que ella está supeditada a la organización política (Pol. 1332b15-16), pero mediante la segunda alusión sugiere que la subsistencia de la organización política depende de aquella (Pol. 1334a9-10). Dada la unidad que para Aristóteles existe entre política, ética y educación, no tiene sentido intentar establecer si la política determina a la educación o a la inversa, puesto que existe una interrelación mutua entre cada uno de estos ámbitos.

18 En Pol. III 4 Aristóteles se ocupa de esta cuestión, pero su respuesta parece ambigua. No obstante, hacia el final del capítulo establece que en un régimen de dominación política la virtud del hombre mejor y la del ciudadano convergen en la figura del gobernante, lo cual ratifica en Pol. III 5 1278a40-b5; 18 1288b37-39. Según KRAUT (1997, 137-138), la identidad entre el ciudadano, el gobernante y el hombre bueno solo es verdadera en el caso de la ciudad ideal, y aclara que ser un buen ciudadano de una oligarquía no implica ser una mala persona, sino que simplemente no es suficiente para la virtud perfecta. 
el fundamento de toda organización política. ${ }^{19}$ Asimismo, este pasaje revela la singularidad de la función que le atribuye al legislador, puesto que — como se evidencia a propósito de la educación musical - su labor coincide con la del filósofo en estas cuestiones (Pol. 1341a31-32). ${ }^{20}$

La división del alma es la respuesta que -sin transición- Aristóteles ofrece a estas preguntas. ${ }^{21}$ A pesar de que no la menciona en Pol. VII 1-3, es el punto de partida del análisis de la educación que presenta en la segunda parte de Pol. VII 14.22 Ella junto con la división análoga de la vida que luego propone son los principios filosóficos que guían la labor legislativa del político (Pol. 1333a3739) y determinan los objetivos de la educación (toùs skopoùs, Pol. 1333b3). ${ }^{23}$ Aun cuando el Estagirita no deja dudas acerca de la superioridad de la parte racional del alma, existe un amplio debate académico sobre la relación entre la razón práctica y la teórica, pues a pesar de los fuertes indicios que abonan un vínculo jerárquico entre ambas, el texto es ambiguo. ${ }^{24} \mathrm{El}$ conocido principio

19 En contraposición a la mayoría de los intérpretes que analizan de manera independiente la segunda parte del capítulo 14, creo -como BÉNatovïL $(2011,162)$ - que la ética no toma aquí el relevo de la política, sino que, por el contrario, las tesis políticas de la primera parte constituyen el fundamento de los análisis psicológicos, éticos y políticos que siguen a ella.

20 Sobre este punto, cfr. Suñol (2014, 317-319).

21 En EN I 13 1102a18-23 Aristóteles afirma que el político debe conocer en cierto modo (pós) las cosas referidas al alma y aclara que debe hacerlo en la medida suficiente para lo que buscamos. La división que allí propone difiere de la de Pol. VII 14, pues incluye la distinción entre la parte vegetativa y apetitiva del alma, pero no alude a la diferencia entre razón práctica y teórica.

22 Kraut $(1997,138)$ asegura que Aristóteles comienza con esta división del alma porque a partir de ella pueden organizarse las distintas etapas de la educación. A mi entender, dicha división es el punto de partida porque la jerarquía de sus partes (toúton dè en potéroi mâllon tò télos, Pol. 1333a19-20) determina la elección de las acciones (Pol.1333a26-30) y permite establecer los objetivos de la educación. La relación entre las partes del alma y las etapas de la educación no se pone de manifiesto aquí, sino en Pol. VII 15. Véase, n. 33.

23 Lisi $(2004,113)$ advierte que en este pasaje Aristóteles refiere a los principios básicos que deben orientar la educación y no a sus contenidos, pues ella es una propedéutica de la vida política e intelectual.

24 Luego de introducir la distinción entre razón práctica y teórica, Aristóteles sugiere que entre ambas hay una jerarquía semejante a la que existe entre la parte irracional y la racional del alma: "Y de igual modo debe dividirse esta parte. Y con respecto a sus acciones, diremos que existe una situación análoga, y aquellas que corresponden a la parte que es mejor por naturaleza deben ser preferidas por los que pueden llevar acabo o bien todas o bien las dos (toîn duoîn), pues siempre para cada uno, lo más deseable es lo más alto que puede alcanzarse." Pol. 1333a26-30. La mayoría de los intérpretes considera que aquí hay una jerarquía, v.gr. Lisi $(2004,101 ; 113)$, Kraut $(1997,138)$, y que a través del dual, refiere a las dos partes inferiores del alma. Por el contrario, Bertelli (2004, 197 n.2) y Demont (1993, 219 notas 28, 30) niegan el carácter jerárquico de esta segunda división y entienden que Aristóteles alude a las partes superiores. Por su 
teleológico que se desprende de la división de la vida permite identificar los fines de la educación, ${ }^{25}$ a saber, ocio (scholêe), paz y acciones nobles (tà kalâ), y además reconocer que el trabajo (ascholía), la guerra y las acciones necesarias y útiles son condiciones e incluso, instancias formativas para ellos (Pol. 1333a30-b5). ${ }^{26}$ Significativamente, Aristóteles sugiere que la educación no está destinada solo a los niños, sino también a los adultos que requieren de ella, con lo cual deja abierta la posibilidad de que ella se prolongue más allá del programa pedagógico propuesto, el cual abarca hasta los veintiún años. ${ }^{27}$ Finalmente, la crítica al belicismo espartano ilustra la estrecha conexión que para el Estagirita existe entre la educación y el régimen político, pues atribuye al legislador la responsabilidad de haber educado a los lacedemonios solo para las virtudes utilitarias y no para ser capaces de disfrutar del ocio (Pol. 1334a6-10), lo cual impide que esta clase de regímenes perviva en tiempos de paz.

parte, BÉNATOUïL (20 I I I I64-I67), quien efectúa un detallado análisis de este pasaje, sugiere que puede haber una jerarquía entre las dos sub-partes racionales, pero que no es necesario hacer como si ella estuviera presente en este pasaje, pues Aristóteles podría haberla dejado voluntariamente implícita para no justificarla. Por esta razón, sostiene que el dual solo puede designar dos actividades entre las cuales el hombre puede elegir, aludiendo así a todas las elecciones limitadas posibles, i.e. entre la primera parte y la segunda, la segunda y la tercera e incluso, entre la primera y la tercera. Según el autor, "Cette interprétation possede a noveau l'avantage supplémentaire de laisser implicite, comme l'a voulu Aristote, la hiérarchie précise entre les deux activités rationnelles." Ibid., p. 167. Las cursivas son mías.

${ }_{25}$ Aristóteles enuncia el principio teleológico en Pol. VII 14 1333a35-36 y en VII 15 1334a15-16. También alude a él en EN 1177b5-6. Respecto a la primera parte del principio, Platón es quien, por primera vez, afirma que la guerra es con vistas a la paz (República 373e5; Leyes 625e-c; 628d-e; 803d4-7; 829a-b).

26 BÉNATOuïL (2011) hace un análisis muy detallado de la conocida tesis teleológica aristotélica con el propósito de esclarecer sus implicancias prácticas. Acertadamente, advierte que Aristóteles no traza una correspondencia directa entre la división del alma y la de la vida, sino que establece una analogía simplemente a nivel de la elección. Asimismo, señala que ambas no son independientes de la división política entre gobernantes y gobernados, y que a pesar de la implícita unidad orgánica que atraviesa el capítulo y de algunas correspondencias que se pueden establecer, las tres divisiones no se superponen. Ibid., pp. 167-171. Sobre la relación que para el autor existe entre los términos de la tesis, véase n. 29.

27 Lord $(1982,34)$ sostiene que Aristóteles propone un programa de educación continua que no se limita a los jóvenes, sino que comprende también a los adultos. Por el contrario, para FORD (2004, 326 n. 50) la idea de la educación adulta es ilusoria. Para una síntesis de las distintas interpretaciones sobre esta cuestión, cfr. Ibid., pp. 328 n. 61. 
El análisis de las virtudes del hombre y del régimen mejor que Aristóteles presenta en la primera parte de Pol. VII 15 (1334a22-25) revela que la filosofía no es la única virtud necesaria para el ocio ${ }^{28}$ sino que se requieren también las virtudes que corresponden al trabajo, i.e. valentía y resistencia (andreías kai karteias), y aquellas que son precisas para ambos momentos, i.e. moderación y justicia (sophrosúnes de kai dikaiosúnes). Como vimos, el trabajo junto con la guerra son aspectos constitutivos en la construcción de un régimen pacífico, cuyo fin último es el ocio. De hecho, desde el punto de vista pedagógico, las actividades no ociosas son fases preparatorias para el ocio y no meros obstáculos o constricciones de las que es preciso librarse (BÉnatouïl 2011, 171-176). ${ }^{29}$ En la segunda parte del capítulo, Aristóteles retoma el tema de la educación, en particular, el orden en que esta debe organizarse. ${ }^{30}$ En primer lugar, destaca que la generación y la práctica de los hábitos debe preceder a la generación y a la práctica de la razón (Pol. 1334b15-17). Por medio de la triple correspondencia que establece entre el cuerpo y el alma, la parte irracional y la racional de esta última, el deseo y la inteligencia como sus estados respectivos muestra la necesidad de que la educación siga el orden de la generación y la jerarquía que -según afirma- subyacen a esta analogía, es decir, que el cuerpo se cuide antes de y con vistas al alma, ${ }^{31}$ el deseo luego de aquel y en pos de la inteligencia (Pol. 1334b1720). ${ }^{32}$ Aun cuando expresamente admite que la razón (ho lógos) y la inteligencia

28 La interpretación que se le otorgue a este empleo de la palabra philosophía es decisiva para la discusión que Aristóteles plantea en Pol. VII 1-3 sobre la vida más elegible. De hecho, existe un amplio debate sobre el significado que aquí tiene el término. La mayoría de los intérpretes lo entiende en sentido amplio referido, de manera general, a las actividades culturales, v.gr. Lord (1978, 354-355); Kraut (1997, 140, 143); Gastaldi (2003, 176); Bertelli (2004, 198). También hay quienes consideran que Aristóteles alude aquí a la filosofía en sentido estricto, v.gr. Lisi $(2004,115$ n.1). Para DePEW $(1991,371)$ el término designaría un ascenso desde la práctica musical a la sabiduría práctica y a la contemplación. Para un análisis más detenido de esta cuestión, cfr. Suñol (2014, 314 n. 47 y 48).

29 BÉNATOUÏL reconoce una relación positiva y no meramente instrumental entre los términos de la tesis teleológica, de modo que el trabajo, la guerra y las acciones necesarias no son meras constricciones de las que es preciso librarse, sino instancias necesarias y preparatorias para el ocio, la paz y las acciones nobles, respectivamente. En este sentido, señala: "L'absence de loisir n'est pas seulement un état que l' on est constraint de subir pour obtener ou avant d'obtenir le loisir, c'est aussi un état requis par le loisir, puisque les vertus formées et exercées dans cet état son útiles non pas seulement pour cet état mais aussi pour le loisir lui-meme.” Ibid., p. 174. Las cursivas son mías.

30 Al igual que en Pol. VII 13, Aristóteles refiere nuevamente en Pol. 1334b6-7 a los tres medios de la educación: naturaleza, hábitos y razón. Véase n. 13.

31 Sobre las dificultades que implican estas distinciones, cfr. Kraut $(1997,147)$.

32 Es verdaderamente aquí y no en Pol. VII 13 -como afirma Kraut $(1997,148)$ - donde se evidencia que la división del alma tiene como finalidad establecer el orden de la educación. Véase 
(ho nôेs) son el fin de la naturaleza humana (Pol. 1334b15), el pasaje revela la necesidad de que la educación del mejor régimen comprenda y atienda todos los aspectos que constituyen al hombre. Así como es preciso fomentar y ejercitar las distintas virtudes del hombre de bien que es, a su vez, el mejor ciudadano, así también la educación debe apuntar a su desarrollo general, pues no es posible construir el régimen mejor si sus ciudadanos son capaces de disfrutar del ocio, pero carecen de valentía en la guerra o a la inversa. Tal es el caso de Lacedemonia, al que de manera recurrente Aristóteles alude, porque es un régimen en el que solo se cultiva una de las virtudes, (Pol. 1334a40-b4), ${ }^{33}$ ya que su fin último es la dominación despótica y la guerra, lo que lo convierte en la contrapartida política y pedagógica de la propuesta aristotélica. En definitiva, la educación de los ciudadanos del mejor régimen no puede centrarse en un aspecto en desmedro de otro, sino que debe ser integral. Las restantes consideraciones que sobre la educación expone hasta terminar el tratado se corresponden con el orden pedagógico aquí propuesto. ${ }^{34}$

En primer lugar, el legislador debe ocuparse de que los futuros ciudadanos tengan un cuerpo acorde al mejor régimen, i.e. que sea resistente al ejercicio sin llegar a ser atlético y con vistas a las actividades propias de los hombres libres (Pol. 1335b2-12). Aristóteles entiende que la constitución física de los ciudadanos comienza a formarse antes de su concepción, de ahí que sus prescripciones regulen desde la edad de los padres (Pol.1334b38-1335a35), el momento más propicio para la unión conyugal (Pol.1335a36-1335b2), las actividades de la madre durante el embarazo (Pol.1335b12-19), los límites cronológicos e incluso intelectuales de la procreación (Pol. 1335b28-37) hasta las condiciones para el aborto (Pol. 1335b22-26). Asimismo, el esbozo del tema que presenta en Pol. VII 16 revela que concibe a la procreación como un servicio (leitourgeîn) ${ }^{35}$ que los ciudadanos deben prestar a la comunidad (Pol. 1335b28-29) y, sobre todo, que su propia

\section{n. 23.}

33 En Pol. VIII 4 1338b11-14 critica a los lacedemonios por considerar que fomentan la valentía a través de los trabajos agotadores, pero -según Aristóteles- estos no propician esa virtud, sino que solo logran transformar a los ciudadanos en brutos.

34 Conforme a dicho orden, en lo que resta del tratado, Aristóteles se ocupa, en primer lugar, del cuidado prenatal del cuerpo (Pol. VII 16), continúa con la instauración de hábitos en la primera infancia, i.e. desde el nacimiento hasta los siete ańos (PolVII 17), luego presenta las distintas disciplinas y etapas de la formación de niños y jóvenes (Pol. VIII 1-2, 4) y culmina con la educación ociosa a través de la música (Pol. VIII 3, 5 y 7).

35 El verbo leitourgéo (Pol. VII 16 1335b28) refiere, de manera general, al servicio al Estado $\mathrm{y}$ al ejercicio de las funciones públicas, s. v. LSJ. 
existencia está supeditada a las necesidades del Estado (Pol.1339b19-26). ${ }^{36}$ Como dejará en claro en Pol. VIII 1, los ciudadanos pertenecen a la pólis antes que a sí mismos.

En Pol. VII 17, se refiere brevemente al cuidado corporal de los nińos a partir de su nacimiento, aludiendo a aspectos que atañen tanto a la alimentación (Pol. 1336a3-8), a los movimientos (Pol. 1336a8-12), como al clima que es beneficioso para la salud y para las actividades guerreras futuras (Pol. 1336a12-21). Siguiendo una tradición de origen pitagórico, Aristóteles divide la educación en tres septenios (Pol. 1336a21-23; 1336b35-1337a1) y, a su vez, distingue tres sub-etapas en el primero, que es cuando los niños son criados en el seno de la familia (Pol. 1336a41-b2). ${ }^{37}$ La primera abarca desde el nacimiento hasta quizás los dos o tres años y, como acabamos de señalar, en esta fase inicial la educación se centra en el cuidado corporal. ${ }^{38}$ Conforme al orden establecido, la segunda de estas subdivisiones, que se prolonga hasta los cinco ańos, apunta no solo al desarrollo físico, si también al del alma, pues además de propiciar el movimiento del cuerpo, está destinada a que los niños se habitúen en el ejercicio de los principios del mejor régimen por medio de juegos, relatos y mitos (Pol. 1336a23-34). Finalmente, de los cinco a los siete ańos comienza la transición hacia la escolarización, ${ }^{39}$ pues devienen espectadores (theoroùs) de los aprendizajes (tôn mathéseọn) que deberán aprender durante el segundo septenio (Pol.1336b35-37), que es cuando -según Aristóteles- debe efectivamente iniciarse la educación pública, si bien el Estado ejerce control en todas las etapas. ${ }^{40}$

36 A propósito de la prescripción aristotélica del infanticidio, KRAUT (1997, 154) asegura: "He does not conceive of individuals as possessors of a natural right not to be killed. Rather, human beings should be protected in various ways only on condition that they are able to make the same contribution to the good of others that is expected of all other full members of the community." Las cursivas son mías.

37 Aristóteles alude a esta división en Pol. VII 16 1335b32-34 y en VII 17 1336b40-1337a1. La importancia que le atribuye aquí y en sus tratados biológicos al número siete revela la influencia pitagórica (HA 553a7, 570a30, 581a12).

38 Aristóteles no especifica hasta cuándo se extiende esta primera etapa (Pol. 1336a21-23).

39 Kraut (1997, 159 ad Gen. Rem.).

40 En este primer septenio la educación transcurre en el seno del hogar y son los padres los responsables de la crianza, estando muy restringida la participación de los esclavos. A pesar de ello, el Estado controla este proceso, tal como lo evidencia la figura del "supervisor de la educación” (paidonómos, Pol.1336a32, a40), magistratura que efectivamente existía en algunas ciudades griegas, como es el caso de Esparta, entre otras. Aristóteles alude también a esta función pública en Pol. IV 15 1299a22-23b y 1300a4. 
En el capítulo hay dos referencias que -a mi entender- son fundamentales para comprender, por un lado, uno de los principales propósitos que tiene la educación de los jóvenes en el régimen ideal y, por otro, cuál es la función que, de manera general, ella cumple en el pensamiento de Aristóteles. En primer lugar, señala la necesidad de prohibir que los jóvenes sean espectadores (theatàs, b20) de yambos y comedias antes de alcanzar su condición de ciudadanos, y aclara que para entonces "la educación <los> hará completamente impasibles (apatheîs) al daño que resulta de tales cosas" (b22-23). ${ }^{41}$ A través de un largo proceso de habituación, la educación logra que a los futuros ciudadanos les resulte extraño lo malo (tà phâula), especialmente, aquello que contenga maldad (mochtherían) u hostilidad (dusméneian) (b34-35). En consecuencia, su función es protegerlos haciendo que no padezcan o sean afectados por todo aquello que contradice los valores del régimen. ${ }^{42} \mathrm{La}$ segunda referencia aparece hacia el final del capítulo, cuando reitera la importancia de organizar la educación siguiendo la división natural, y aclara "ya que todo arte (téchne ) y educación (paideía) tienden a completar lo que falta de la naturaleza" (Pol. 1337a1-3). ${ }^{43}$ A pesar de la concisión con la que se expresa, Aristóteles explicita aquí uno de los principales supuestos de su pensamiento: las artes y la educación son complementarias a la naturaleza, pues ambas resultan indispensables para la supervivencia y el desarrollo humano. ${ }^{44}$ Precisamente, esto evidencia la importancia capital que para el filósofo tiene la educación y que los intérpretes parecen no reconocer de manera suficiente.

41 La primera de estas alusiones aparece en el contexto de las estrictas prohibiciones que establece en cuanto a la exposición de los nińos y jóvenes del mejor régimen a todo aquello que signifique una degradación moral, ya sea el lenguaje vergonzoso (aischrologían, 1336b4), el contemplar (tò theoreîn) representaciones o discursos inconvenientes/indecentes (aschémonas, b14), la existencia misma de estatuas o dibujos que imiten actos de tal clase (b15-16), llegando incluso a imponer castigos acordes a la edad para quienes no se atengan a ellas (b8-12). Solamente admite la presencia de esta clase de representaciones en los templos de los dioses para los que la ley admite la burla (tòn tôthasmòn, b17), razón por la cual restringe su culto a los varones adultos (b18).

${ }^{42}$ Top. 125b22-27.

43 Aunque Aristóteles las distingue, podemos preguntarnos si acaso la educación no puede ser subsumida en la categoría de las artes o si como afirma Lisi se trata de una propedéutica. Véase n. 24.

44 Aristóteles alude al carácter complementario que las artes tienen respecto de la naturaleza en Protréptico B13 y en Física II 8 (199a15-17). Para un análisis detallado de esta cuestión, cfr. Suñol $(2012,175-184 ; 194-195)$. 


\section{La educación como fundamento de todo régimen político}

El último libro de la Política está enteramente dedicado a la educación de los jóvenes en el régimen ideal. A pesar de su relevancia, ha merecido relativamente poca atención por parte de la literatura especializada, porque es considerado "una suerte de apéndice o pensamientos adicionales sobre un tema menor" (DestrÉE, 2013, 301). ${ }^{45}$ Sin embargo, a lo largo de Pol. VIII Aristóteles no solo describe -si bien de manera esquemática e incompleta- los aspectos vinculados a la educación pública durante el segundo y tercer septenio, con especial atención a la mousiké, que es la disciplina que fundamenta el programa educativo ideal, sino que también explica la importancia que la educación, en general, tiene para todo régimen político. De hecho, hacia el final de Pol. VII 17 replantea tres cuestiones básicas referidas al tema: en primer lugar, pregunta si es necesario establecer alguna disposición con respecto a los niños, segundo, si su cuidado debe tener carácter público o privado y, finalmente, cómo debe organizarse (Pol. 1337a3-7), a las cuales responde detenidamente en los dos primeros capítulos del libro VIII. ${ }^{46} \mathrm{Al}$ comienzo de Pol. VIII 1, contesta al primer interrogante afirmando que la educación de los jóvenes debe ser la principal ocupación de quien establece las leyes, i.e. el legislador (nomothéthes).$^{47}$ Aunque lo plantea como un hecho indiscutible, reconoce que este interés primario por la educación no se da efectivamente en todas las ciudades y admite que en esos casos, se daña el régimen político (Pol. 1337a12-14). El carácter (êtthos) de cada organización política es el fundamento sobre el cual cada una se construye y que, a su vez, permite preservarla. ${ }^{48}$ Mediante su programa pedagógico, procura propiciar el mejor carácter para el mejor

45 "[...] no more than a sort of appendix or additional thoughts on a minor subject."

46 Como advierte Kraut (1997, 168 ad 37a3-7), resulta extraño que Aristóteles plantee estas preguntas en este punto, cuando ya se ha dedicado ampliamente al tema. El autor sugiere que quizás retoma el tema aquí porque está por proponer una ruptura radical con la práctica contemporánea: "[...] he sees the need to return to more fundamental considerations, in order to win acceptance of his proposal for public education".

47 Como se consignó en la nota anterior, Aristóteles de manera implícita ha dado una respuesta afirmativa a esta cuestión en el tratamiento que viene dando al tema desde que inicia sus consideraciones sobre la educación en Pol. VII 13. Según Kraut (1997, 169), los libros VII y VIII conforman un único tratado y su división es artificial. Asimismo, asegura que al comienzo del libro VIII Aristóteles hace una serie de reflexiones generales sobre la educación, porque es en este punto donde debe ocuparse de la formación de los niños a partir de los siete años, que es cuando ésta pasa a ser responsabilidad de la ciudad. Quizás esa sea la razón por la cual en el final de Pol. VII 17 refiere a los niños (toùs paîdas, 1337a4) y al comienzo de Pol. VIII 1 habla de los jóvenes (tônn néon, Pol. 1337a12).

48 Platón refiere a la correspondencia entre los caracteres humanos y los regímenes políticos en República 544d. 
régimen, lo cual requiere que los jóvenes hayan sido educados (propaideúesthai) y acostumbrados (proethizesthai) para la práctica de la virtud (Pol. 1337a17-21). ${ }^{49}$

En lo que resta del capítulo (Pol. 1337a21-32), Aristóteles responde al segundo de los interrogantes planteados en Pol. VII 17 (1337a4-6), referido a si el cuidado de la educación debe ser público o privado, como era práctica habitual en muchas ciudades. ${ }^{50}$ De manera muy sucinta pero contundente, explica que la educación debe ser una y la misma para todos los ciudadanos porque uno es el fin del régimen (1337a21-23), el cual sabemos por lo dicho a lo largo del libro VII, que es la eudaimonía entendida como el ejercicio de la virtud. ${ }^{51}$ En consecuencia, la unidad y homogeneidad del programa educativo está determinada por la organización teleológica del régimen político. Si bien el programa educativo que diseña responde al interés político de construir el mejor régimen, las consideraciones sobre la educación que expone en Pol. VIII 1-2 tienen un carácter general, pues se aplican a todas las formas de gobierno, ${ }^{52}$ aunque no deja de destacar la superioridad de aquella forma de organización política (aei dè tò béltion êthos beltionos aition politeías, Pol. 1337a17-18). Al postular la uniformidad del

49 En otros pasajes, Aristóteles subraya la importancia de educar a todos los miembros de la ciudad conforme al régimen político en el que viven. Así por ejemplo, en Pol. I $131260 \mathrm{~b} 13$ se refiere a la educación de los hijos y las mujeres que componen la casa, cuya virtud es preciso cultivar con vistas a la del todo que componen. También en Pol. V 9 1310a12-18 hace una alusión general a la cuestión.

50 Aunque Aristóteles critica frecuentemente el carácter militarista de la educación espartana (v. gr. Pol. VII 2 1324b6-9), en Pol. VIII 1 1337a31-31 elogia la atención que los lacedemonios le dedican a los niños y el hecho de que hacen de ello una cuestión comunitaria. En EN X 9 1180a24-28 hace una consideración semejante sobre el carácter público de la educación espartana, en contraste con la costumbre predominante en la mayoría de las ciudades.

51 Los argumentos del libro VII permiten afirmar que el fin al que Aristóteles alude es la eudaimonía (Pol. VII 2 1324a5-7; 13 1331b39-40), a la cual entiende estrechamente ligada a la virtud (Pol. VII 1 1323b21-23, 1323b40-1324a2; VII 3 1325a16-17; Pol. VII 8 1328a38; Pol. VII 9 1328b36, 1329a22-23; Pol. VII 13 1332a9). En Pol. VII-VIII el eje de la discusión se centra en las formas de vida y no en el significado de la eudaimonía, si bien esta es el fin de la vida individual y comunitaria. En consecuencia, ambas cuestiones están íntimamente ligadas y son, en cierto modo, inseparables. Aristóteles asegura que los defensores de ambos bioi coinciden en cuanto a que el fin es la virtud, pero discuten si ella comporta o no la actividad política (Pol. VII 2 1324a25-35). A través del régimen de alternancia, que establece como forma de garantizar un dominio político y no despótico, y de la pertenencia comunitaria de todos los ciudadanos, no deja dudas respecto a que la virtud que conduce a la eudaimonía conlleva la participación política. Sobre esta cuestión, cfr. SuÑol (2014).

52 Este carácter general se evidencia cuando afirma que en las ciudades en las que el legislador no se ocupa de la educación se daña el régimen (Pol. 1337a12-14) y cuando sostiene que el fin es uno en todas las ciudades (Pol. 1337a 21-22). 
programa educativo, el Estagirita parece no tener en cuenta las singularidades de los ciudadanos que conforman un régimen político, ni siquiera las de aquellos que connaturalmente son superiores al resto. ${ }^{53}$ De ahí que contradiciendo la costumbre de la época y a diferencia de lo que hace su maestro en la República al formular un programa específico para los guardianes-filósofos, ${ }^{54}$ establece un único programa educativo para todos y cada uno de los ciudadanos, y de responsabilidad estatal. ${ }^{55}$ La homogeneidad del programa educativo -que, como vimos, está determinada por la unidad del fin a la que toda ciudad apunta- supone que todos los ciudadanos gracias a la igualdad de la educación pública que reciben están involucrados en la persecución de ese fin comunitario. ${ }^{56}$

53 En Pol. VII 12 Aristóteles refiere a la existencia en el régimen ideal de una gran variedad de magistraturas - las encargadas del ágora (agaronomían, 1331b9), de la ciudad (astunomían, 1331b10) y del territorio (buloroùs, agronomoús, 1331b15) - e incluso reconoce una jerarquía entre ellas (tà kupiótata tôn archeión, Pol. VII 12 1331a25), la cual se refleja en la disposición de los edificios de los templos, los de las comidas comunes, los gimnasios y, sobre todo, la distinción que establece entre el ágora libre y la necesaria. A la luz de ello, la homogeneidad del programa educativo ideal no supondría negar las diferencias y singularidades de los ciudadanos, en la medida en que cada uno desempeñaría estas diversas responsabilidades en función de sus características particulares, pues difícilmente todos los ciudadanos podrían ejercer todas las magistraturas a lo largo de sus vidas.

54 LIsI $(2004,115$ n.1) afirma que el carácter inconcluso del libro VIII nos impide saber si establecía un programa educativo especial para los que naturalmente han sido dotados para esta actividad, pero Aristóteles expresamente destaca la unidad y uniformidad del programa educativo ideal, por lo cual difícilmente podemos aceptar esa posibilidad. Por su parte, KraUt $(1997,139)$ sostiene que Aristóteles no hace referencia a un curso de estudios especiales para que los ciudadanos devengan filósofos porque no todos son capaces de alcanzar la excelencia teorética y quienes sí pueden hacerlo no requieren de entrenamiento cívico especial, sino que lo harán naturalmente. Dado su carácter excepcional, la filosofía no es, según KRAUT, el propósito para el cual deben ser educados los ciudadanos del régimen ideal, aun cuando reconoce que seguramente Aristóteles creía que esta es la mejor forma de vida. En este mismo sentido, admite la posibilidad de que el Estagirita concibiera a la educación de todos los ciudadanos como una preparación para una actividad que está próxima a la de los filósofos y ello explicaría la gran atención que le dedica a la educación musical. A mi entender, el programa educativo ideal apunta a que todos los ciudadanos e incluso habitantes de la pólis sean capaces de participar de las actividades ociosas, las cuales comprenden desde la formas más vulgares de la música hasta la filosofía en sentido estricto.

55 Aristóteles afirma en Pol. II 7 1266b29-38 que la uniformidad educativa no garantiza por sí misma que la educación sea buena, sino más bien el contenido del programa y el propósito al que apunta. Tampoco responde a una pretensión igualitaria por parte del filósofo: "[...] but his aim is not to minimize or eliminate the effect of differences in wealth" KraUt (1997, 170).

56 Aristóteles no afirma aquí que todos los ciudadanos deben perseguir un único objetivo, pero la estrecha conexión que establece entre el fin del individuo, el de la ciudad (Pol. VII 2 1324a5-13) y la uniformidad del programa educativo nos lleva a pensar que todos apuntan al mismo. Por esta razón, el programa educativo propuesto no atiende a las distintas capaci- 
El carácter comunitario que debe tener la educación está determinado por un supuesto más amplio sobre la relación de los individuos con el Estado, el cual Aristóteles reconoce de manera expresa cuando afirma que "no es preciso considerar que entre los ciudadanos, alguno de estos es para sí, sino que todos <son> de la ciudad, pues, cada uno es parte de la ciudad" (Pol. 1337a27-29). ${ }^{57}$ Todos los ciudadanos sin excepción pertenecen a la pólis y, por ende, todos están de igual forma comprometidos con el fin de esta, independientemente de sus distintas aptitudes y capacidades. Esto no implica que Aristóteles niegue estas diferencias, sino que las incluye como parte de un todo, pues asegura que "el cuidado de cada una de las partes apunta naturalmente al cuidado del todo" (Pol. 1337a30). ${ }^{58} \mathrm{Al}$ admitir que el ciudadano pertenece primariamente a la comunidad política antes que a sí mismo, nos ofrece una pieza clave para comprender su pensamiento ético-político-pedagógico. El pasaje refleja el modo en que el Estagirita, en particular, y los griegos, en general, concebían la relación con el Estado, pues todas las actividades de los ciudadanos desde el matrimonio, las uniones sexuales (Pol. VII 16), el ejercicio de las funciones militares, cívicas y religiosas (Pol. VII 9), el cuidado pre y post-natal de los niños (Pol. VII 16, 17) y, de manera integral, su educación (Pol. VII 17, VIII) eran concebidos como servicios públicos que estos debían prestar a la pólis, en cuanto partes constitutivas de ella. Como advierte Kraut $(1997,173)$, esto revela la distancia que existe con nuestra concepción política moderna. La pertenencia al Estado no era el resultado de una elección racional por parte de los ciudadanos, sino que la existencia de este era conceptualmente anterior a la de sus miembros, ${ }^{59} \mathrm{y}$ formaba

dades de los ciudadanos, porque todos los miembros de la pólis están, en la medida de sus posibilidades, comprometidos con dicho fin comunitario.

57 Platón hace referencia a la importancia de la educación pública y obligatoria y a la pertenencia de los ciudadanos al Estado más que a sus padres (họs tệs póleọs mâllon $\underline{e}$ tôn gennétóron óntas) en Leyes $804 \mathrm{~d} 5$. Sobre la pertenencia comunitaria, también hace alusión en Leyes $923 \mathrm{a} 8-\mathrm{b} 1$.

58 En Pol. VII 8 y 9 Aristóteles distingue las partes de la pólis de sus elementos constitutivos. Solamente los ciudadanos, quienes de manera alternada o permanente se dedican a las actividades militares, deliberativas o sacerdotales, son parte de ella y, por ende, integran ese todo. Por el contrario, los agricultores, artesanos y jornaleros son condiciones necesarias para la existencia de la pólis, pero no forman parte de ella (Pol. VII 9 1329a34-39). Tanto los ciudadanos como los habitantes garantizan la autosuficiencia de la ciudad (Pol. VII 8 1328b1519), aun cuando el principio sobre la relación de las partes con el todo que formula en Pol. VIII 1 solo se aplica a los primeros. En Leyes 903b-d Platón hace referencia a la relación de las partes con el todo, pero desde una perspectiva universal y no cívica.

59 Aristóteles se refiere a que el todo, i.e. la pólis, es por naturaleza necesariamente anterior a las partes, i.e. los individuos y la casa, en Pol. I 2 (1253a18-20). El hecho de que reconozca que históricamente alguien fundó por primera vez una ciudad (1253a30-31), revela 
parte de su naturaleza, ${ }^{60}$ ya que solo como miembros de una comunidad política estos podían alcanzar el fin último del individuo y, por ende, de la comunidad. ${ }^{61}$

En Pol. VIII 2 Aristóteles responde al tercero de los interrogantes planteados al final del libro VII (Pol. 1337a6-7), el cual refiere al modo en que debe organizarse la educación y, de manera más amplia, también se pregunta por su naturaleza (tís d'éstai he paideía kaì pộs chrè paideúesthai, Pol.1337a 34-35). En primer lugar, hace alusión a las controversias que, por entonces, existían en torno a este tema (Pol. 1337a35-36). ${ }^{62}$ De sus afirmaciones, se desprende que la educación depende de cómo se define la virtud ${ }^{63}$ y de cuál se considere que es la vida mejor (Pol. 1337a36-38). Este pasaje ilustra claramente que el análisis de la educación es inseparable de la consideración de las cuestiones éticas más importantes. En este mismo sentido, reconoce que tampoco hay acuerdo respecto a su propósito: si es más adecuado organizarla con vistas al entendimiento (pròs tèn diánoian) o al carácter del alma (pròs tò tệs phuchệs êthos, Pol. 1337a38-39). ${ }^{64}$ Esta tensión entre intelecto y carácter remite al debate dialéctico que el Estagirita establece en los capítulos del prólogo dedicados a elucidar la vida mejor (Pol. VII 1-3), lo cual sugiere la correspondencia que para el filósofo existe entre el modelo pedagógico que se adopte y la elección de la forma de vida, i.e. entre paideía y bios. Asimismo, sostiene que la educación vigente tampoco permite aclarar el tema, ya que no hay acuerdo respecto a si se deben ejercitar las cosas útiles para la vida o las que apuntan a la virtud o incluso, las extraordinarias ( $t a ̀$ perittá, 1337b42), y aclara que cada una de ellas ha recibido juicios favorables. ${ }^{65}$

que no está pensando en una anterioridad cronológica de la ciudad con relación a los individuos.

$60 \mathrm{Al}$ respecto, me limito a mencionar dos pasajes paradigmáticos: "el hombre es por naturaleza un animal político", Pol. I 2 1253a2-3 y "el impulso hacia esa clase de comunidad está en todos por naturaleza", a29-30.

${ }_{61}$ "Pero no entendemos por suficiencia el vivir para sí solo una vida solitaria, sino también para los padres y los hijos y la mujer y en general para los amigos y ciudadanos, porque el hombre es por naturaleza político" EN I 7 1097b8-11.

62 "Ahora se discute acerca de estos temas <sobre la educación>. Pues, no todos asumen que es necesario que los jóvenes aprendan las mismas cosas ni respecto a la virtud ni a la vida mejor."

63 Unas líneas más adelante, Aristóteles advierte que tampoco hay acuerdo respecto al ejercicio de la virtud, porque "simplemente no todos valoran la misma virtud" (Pol. 1337b1-3).

64 "His assumption that education of the character does not sufficiently educate the mind may rest on his belief that a philosophical life, or more generally speaking, an intellectual life, is better than a life that exercises only the virtues of character." KraUt $(1997,175)$.

65 Inmediatamente a continuación de esta afirmación, Aristóteles reconoce que es indispensable que los ciudadanos conozcan las cosas útiles y a lo largo, de todo su análisis de la educación 
Aristóteles dedica lo que resta de Pol. VIII 2 a analizar la naturaleza de los conocimientos que deben enseñarse. En este punto, introduce la distinción entre las artes liberales e iliberales (1337b5-6), ${ }^{66}$ define qué es lo que entiende por vulgar (bánauson, b8), ${ }^{67}$ establece restricciones sobre el ejercicio de las artes liberales (b15-17) y, finalmente, destaca la importancia de la finalidad por la cual algo se realiza o aprende (b17-23). Si bien reconoce que deben enseñarse las cosas útiles que son necesarias, advierte que solo las que no hagan vulgares a los hombres que participan de ellas. ${ }^{68}$ Todo aquello que atenta contra el ejercicio corporal e intelectual de la virtud define el carácter banausico de las actividades, artes y enseñanzas. Por esta razón, considera que las actividades asalariadas pueden ser incluidas en esta categoría, porque privan de ocio al entendimiento (diánoian) y lo degradan (b14-15).

señala la importancia de que esta apunte a la virtud, pero nunca aclara a qué refiere con "las cosas extraordinarias". Esta misma expresión es la que emplea en EN VI 7 1141b3-9 para referir al conocimiento de Anaxágoras y de Thales, de quienes se dice que saben cosas extraordinarias, admirables, difíciles y divinas, pero inútiles porque no buscan los bienes humanos. Como advierte KraUt $(1997,176)$ : "he is probably classifying astronomy, geometry, and other scientific subjects as out of the ordinary."

${ }^{66}$ Aristóteles no fue el primer pensador griego que discute las artes liberales, ya que el tema circulaba en la Atenas del siglo V a.C. Sin embargo, Nightingale (1996, 29) sostiene que su discurso puede ser considerado fundacional, en la medida en que es la única explicación del tema del período clásico que está completamente conceptualizada y articulada.

67 De manera general, este término designaba a la gente que ganaba su existencia a través de la ejecución de un arte que involucraba el empleo de las manos, y era virtualmente empleado como sinónimo de anaeleútheros. Nightingale (i996, 30-34) advierte que no se trata de una palabra simplemente descriptiva, sino que refleja la perspectiva y los prejuicios de la elite ociosa, ya que se sustenta en la ideología aristocrática. Asimismo, señala que durante el período clásico el término fue monopolizado por Platón, Jenofonte y Aristóteles, y no aparece atestiguado ni en textos de comedia, ni de oratoria.

68 Nightingale $(1996,35-39)$ sostiene que en Pol. VIII hay dos perspectivas de la educación liberal y que ambas refieren a propósitos diversos. La primera entiende a la educación como el medio que posibilita que los ciudadanos se hagan virtuosos. La otra -que Aristóteles introduciría en Pol. VIII 2- destaca que la educación liberal es perseguida por sí misma y no tiene ningún otro propósito y ello se vincula a su concepción de la libertad. Además, la autora identifica dos clases de hombres libres en la Politica, a saber: los que ejercen el dominio político y quienes se dedican a la contemplación, que es la forma más radical de la libertad. La educación liberal, asegura, apuntaría a propiciar ambas formas de libertad, al producir ciudadanos y gobernantes virtuosos que reconozcan que la actividad primaria del sistema educativo debe ser practicada por sí misma. A su juicio, Aristóteles fue el primero en destacar la inutilidad de la filosofía, que es la única actividad verdaderamente ociosa y advierte que ella no es intrínsecamente inútil ni improductiva, sino que es el propio Estagirita quien le atribuye este rasgo. A decir verdad, los dos modelos de libertad que la autora dice identificar no están consignados como tales en el texto de Pol. VII-VIII. 
En los restantes capítulos del libro VIII, Aristóteles bosqueja el currículo de la escolaridad pública durante el segundo y tercer septenio, i.e. de los siete a los veintiún años. Pero, principalmente, se ocupa de la mousiké, que es la piedra fundamental del programa educativo del régimen ideal (Nightingale, 1996, 35), pues encarna el modelo de la educación liberal y ociosa. ${ }^{69}$ Aunque no podemos profundizar aquí el estudio de los distintos problemas que subyacen a esta disciplina, $v . g r$. sus distintas funciones, su relación con la filosofía, la división de las melodías propuesta en Pol. VIII 7, etc., es necesario destacar su importancia política en la construcción del régimen ideal. ${ }^{70}$ En efecto, al ser una disciplina cuyo propósito más elevado es el ocio (scholé) ella permite alcanzar el fin del mejor régimen, i. e. la eudaimonía (Pol. 1338a1). ${ }^{71}$ Aristóteles le confiere un carácter socialmente abarcador, pues adjudica la ejecución musical a los asalariados (thêtikotéran, Pol. VIII $61341 \mathrm{~b} 14$ ) y a los jóvenes, mientras que a los ciudadanos adultos les atribuye el juzgar las cosas bellas (tà kalà krinein, 1340b38) así como el disfrutar de ellas correctamente (chareín orthôss, 1340b39), gracias al aprendizaje desarrollado en su juventud (Pol. VIII 6 1340b35-40). El desprecio por las actividades artesanales lo lleva a prohibir que los hombres libres (tôn eleuthéron, 1341b13) reciban la educación profesional de los instrumentos y de la ejecución, i.e. aquella destinada a los concursos, por considerar que atenta contra su virtud al estar supeditada al placer vulgar (phortikêss, b12) de los oyentes y, en general, a la vulgaridad (banáusous, b14) de los espectadores (theatès, b16) (Pol. 1341b8-18)..$^{72}$ A pesar de las restricciones que establece con respecto

69 A pesar de la importancia que le reconoce, Aristóteles no solo no afirma que esta sea la única actividad ociosa a la que deben dedicarse los ciudadanos, sino que incluso se pregunta si la educación liberal de los hijos comprende una o más disciplinas de esta clase, cuestión que deja sin resolver (Kraut 1997, 183; DestréE 2013, 316 n. 15).

70 Estas cuestiones serán específicamente estudiadas en un trabajo, que se encuentra en preparación y en el que profundizaré el estudio de Pol. VIII 3, 5-7.

71 La relevancia que Aristóteles le otorga al ocio como principio único de todo (haúte gàr archè pánton mía, 1337b32) supone la exclusión de las clases trabajadoras de la ciudadanía. No obstante, el ocio está inseparablemente ligado a la construcción de un Estado pacífico, pues junto con la paz y las acciones nobles conforman los pilares de dicho régimen (Pol. VII 141333 b3036). El empleo de esta noción en los dos últimos libros de la Pol. es complejo, pues en Pol. VII 9 1329a 1 presenta al ocio como condición de las acciones políticas y de la virtud, mientras que en el libro VIII no hace ninguna referencia a su vínculo con la política, más aun como señala KRAUT: "[...] he gives every indication of believing that it is unleisurely, since political activity does not figure at all in his discussion of leisure in Pol. VIII" (1997, 179-180). El tema ha dado lugar a interpretaciones contrapuestas, $v$. gr. SOlMSEN (1964) y LORD (1978). Por mi parte, coincido con KRAUT en que Aristóteles deja irresuelta esta cuestión.

72 Esto refleja una crítica por parte del filósofo a la profesionalización y al virtuosismo que al parecer, por entonces, influía en la enseńanza musical (Pol. 1341a9-14). 
a la educación y a la práctica musical, todos los integrantes de la pólis — de una forma u otra- participan del goce musical, el cual tiene un carácter connatural común; aun cuando propicia un placer superior (Pol. VIII 5 1340a2-6; VIII 6 1341a15-17). Por esta razón, Aristóteles propone ritmos, armonías e incluso competencias y espectáculos (agônas kaì theorías, 1341a21) adecuados para cada edad (1342b20), tipo de espectador y propósito al que se apunte: ya sea la educación de los futuros ciudadanos para la virtud política (Pol. VIII 6 1340b421341a1; VIII 7 1342a28-29), sea el descanso (anápausin) de los espectadores vulgares (Pol. VIII 7 1342a19-22), sea la relajación de los ciudadanos en la vejez (Pol. VIII 7 1342b20-23; b27-29) o su educación durante la infancia (Pol. VIII 7 1342b29-33).

\section{Conclusiones}

El rastreo realizado a lo largo del presente trabajo revela que para Aristóteles el estudio de la educación es inseparable de la indagación ética y de la política. En este sentido, puede decirse que las tres forman parte de un único cuerpo de conocimiento, que podría identificarse con lo que el filósofo denomina "ciencia política” (EN 1094a26-b11). ${ }^{73}$ De hecho, ello permite entender por qué no es posible encontrar un tratamiento separado del tema en el corpus y, además, explica el lugar que ocupa en sus tratados ético-políticos más importantes. En efecto, luego de la indagación ética que desarrolla a lo largo de la $E N$ termina destacando, en el último capítulo de la obra, la importancia de la educación para hacer efectivo ese conocimiento (ENX $91179 \mathrm{a} 35-\mathrm{b} 2) ; ;^{74}$ y esta cuestión, a su vez, anticipa la investigación política (EN 1181b12-22). Por otra parte, aunque resulte problemática la ubicación de Pol. VII-VIII en el marco general de la obra, ${ }^{75}$ no hay dudas respecto a que el diseńo del régimen político ideal concluye con la elaboración de un programa pedagógico. La educación constituye el medio que permite hacer efectivo el conocimiento ético-político; de ahí que cada una de estas disciplinas no está completa sin la consideración de la educación. Además, a pesar de los supuestos aristocráticos que subyacen al pensamiento político aristotélico, resulta significativo que haya elaborado un único programa educativo, uniforme para todos los ciudadanos y de carácter

73 Depew (2009, 399-400), Cooper (2010, 230), Suñol (2013, 17, 25 notas 21 y 39, respectivamente).

74 ENI 3 1095a5-6.

75 Newman (1902). 
público. En definitiva, la educación es para Aristóteles el pilar a partir del cual se construye todo régimen político y, en particular, en el caso del mejor régimen requiere de un programa pedagógico integral que se inicia antes del nacimiento e incluso de la concepción de los ciudadanos, que probablemente se prolongue en la edad adulta y en el que estos se educan en el ocio con el propósito último de que lleguen a ser felices en el marco de un Estado pacífico.

\section{Agradecimientos}

Agradezco al Prof. Pierre Destrée por ayudarme a localizar material relevante para la realización del presente trabajo y, sobre todo, por impulsarme, gracias a su estimulante artículo: "Education, Leisure and Politics" (2013), a reconsiderar aspectos centrales de mi investigación.

\section{Bibliografía}

BÉnatouïL, T. (2011). "'Choisir le labeur en vue du loisir': une analyse de Politiques, VII, 14”. En: E. Bermon, V. Laurand, J. Terrel (eds.) Politique d'Aristote: Famille, régimes, éducation. Pessac: Presses Universitaires de Bordeaux.

Bertelli, L. (2004). "L'elogio del bios praktikos in Politica VII di Aristotele". En: Lisi, F. (ed.), The Ways of Life in Classical Political Philosophy, Sank Augustin: Akademie-Verlag, pp. 179-200.

Cooper, J. M. (2010). "Political community and the highest good". En: Lennox, J. G. y Bolton, R. (eds.), Being, Nature and Life in Aristotle. Essays in Honor of Allan Gotthelf, Cambridge: Cambridge University Press, pp. 212-264.

Demont, P. (1990). La cité grecque archaïque et classique et l'idéal de tranquillité, Paris: Belles Lettres.

Depew, D. J. (1991). "Politics, Music and Contemplation in Aristotle's Ideal State". En: Keyt, D. y Miller, F. D. (eds.) A Companion to Aristotle's Politics, Oxford Cambridge, Mass.: Blackwell, pp. 346-380. 
- (2009). "The Ethics of Aristotle's Politics". En: Balot, R. (ed.) A Companion to Greek and Roman Political Thought, New York: Blackwell, pp. 399-418.

Destrée, P. (2013). “Education, leisure, and politics”. En: Deslauriers, M. y Destrée, P. (eds.). The Cambridge Companion to Aristotles Politics. Cambridge: Cambridge University Press, pp. 301-323.

Ford, A. (2004). "Catharsis: The Power of Music in Aristotle's Politics". En: Murray, P. y Wirson, P. (eds.). Music and the Muses. Oxford: Oxford University Press.

Gastaldi, S. (2003). Bios Hairetotatos. Generi di vita e felicita in Aristoteles, Napoli: Bibliopolis.

García Valdés, M. (1988). Aristóteles. Política. Introducción, traducción y notas. Madrid: Gredos.

Hummel, Ch. (1997). "Aristotle”. En: Morsy, Z. (ed.) Thinkers on Education. Oxford \& IBH Publishing/UNESCO-IBE, pp. 1-11.

Disponible en: http://www.ibe.unesco.org/publications/ThinkersPdf/aristote.pdf

JaEger, W. (1993). Paideia: los ideales de la cultura griega. Traducción de Joaquín XIRAU y Wenceslao Roces. México: Fondo de Cultura Económica. (1933¹).

Kraut, R. (1997). Aristotle. Politics. Books 7-8. Traducción y comentario. Oxford: Clarendon Press.

Lisı, F. (2004). "Vida teórica, vida práctica y felicidad en Aristóteles". En: _. (ed.), The Ways of Life in Classical Political Philosophy, Sank Augustin: Akademie-Verlag, pp. 95-120.

Lord, C. (1978). "Politics and Philosophy in Aristotle's Politics", Hermes CVI, pp. 336357.

- (1982). Education and Culture in the Political Thought of Aristotle. Ithaca - Londres: Cornell University Press.

Marrou, H. I. (1948). Historie de l'Éducation dans l'Antiquité. Paris: Éditions du Seuil.

Newman, W. L. (1902). The Politics of Aristotle, with an Introduction, two prefatory essays and notes critical and explanatory, Vol. III, Oxford: Clarendon Press.

Nightingale, A. W. (1996). "Aristotle on the 'Liberal' and 'Illiberal' Arts", Proceedings of the Boston Area Colloquium of Ancient Philosophy 12 (1), pp. 29-58.

Reeve, C. D. C. (1998). "Aristotelian Education". En: A. O. RorTy. (ed.) Philosophers on Education: Historical Perspectives. Oxford: Routledge, pp. 51-65.

Santa Cruz, M. I.; Crespo, M. I. (2005). Aristóteles. Politica. Introducción, traducción y notas. Buenos Aires: Losada. 
Solmsen, F. (1964). "Leisure and play in Aristotle's Ideal State". Rheinisches Museum für Philologie, CVII, 3, pp. 193-220.

Suñol, V. (2012). Más allá del Arte: Mimesis en Aristóteles. La Plata: Universidad Nacional de La Plata.

- (2013) "La discusión aristotélica sobre los modos de vida. El contraste entre el bios theoretetikós en Ética a Nicómaco X 7-8 y el bios praktikós en Politica VII 3”. Tópicos 45, pp. 9-47.

- (2014). "La mejor forma de vida en el régimen político ideal de Aristóteles". Anales del Seminario de Historia de la Filosofía, Vol. 31, No 2, pp. 311-336.

Tachibana, K. (2012). "How Aristotle's Theory of Education Has Been Studied in Our Century”, Studida Classica 3, pp. 21-67.

Enviado: 19/06/2015

Aceptado: 31/07/2015

Este trabajo se encuentra bajo una licencia de Creative Commons ReconocimientoNoComercial-SinObraDerivada 4.0

\section{(c) $\underset{\mathrm{EY} \text { NG } \mathrm{ND}}{\mathrm{N}}$}


